\section{P135 FACTORS THAT INFLUENCE LEARNING OF SEXUAL HISTORY TAKING FOR MEDICAL STUDENTS}

doi:10.1136/sextrans-2012-050601c.135

J Davies.* Royal Sussex County Hospital, Brighton, UK

Background Training for medical students in the UK now incorporates formalised training in sexual health and HIV. While research has assessed knowledge and skills in medical graduates around sexual history taking, this has been largely approached from a quantitative paradigm rather than students own experience It was decided by the author to carry out a qualitative study to inform content and delivery of the current sexual and HIV module.

Aim To explore the experiences of fourth year medical students in their learning of sexual history taking.

Methods Semi-structured interviews were undertaken with six fourth year medical students. Framework analysis was used to identify emerging categories and themes from the data.

Results Four distinct categories were acknowledged: prior experience, classroom based learning, clinic based learning, and future confidence. Themes identified included patient and student embarrassment, acquisition of key phrases, the use of proformas and patient consent. Factors influencing the learning of sexual history taking were often interwoven and stemmed from both the classroom and clinical setting. One common expectation was that another healthcare professional would take over while being observed with a patient. The issue regarding confidence appeared to be intrinsically built up from the start of their overall training. Students recommended that classroom based scenarios should include non-genito-urinary medicine settings. Student confidence was improved by the recognition that classroom teaching matched the clinical consultations, with no preference to designation of the health care professional teaching.

Conclusion Acknowledgement of the influences experienced by medical students while undertaking classroom and clinic based learning of sexual history taking provides useful guidance for future curriculum development.

\section{P136 SEXUAL HEALTH SERVICES HAVE A KEY ROLE IN THE DIAGNOSIS AND INITIAL ASSESSMENT OF HEPATITIS C}

doi:10.1136/sextrans-2012-050601c.136

H Donson, ${ }^{*}$ K E Rogstad, L Campbell, M Raza, A Cope. Sheffield Teaching Hospitals NHS Trust, Sheffield, UK

Background Hepatitis $\mathrm{C}$ is a major cause of liver disease, cirrhosis and liver cancer but is increasingly amenable to treatment. Sexual health services often have unique access to test patients within risk groups.

Aim To assess the role of sexual health services in diagnosis and initial assessment of Hepatitis C (HCV).

Method Patients diagnosed with HCV at a large inner city sexual health service from 1 July 2009 to 30 June 2011 were identified along with a control group of negative patients.

Results $4430 \mathrm{HCV}$ tests were performed on 3395 patients. 53 (1.56\%) were HCV antibody positive of which 26 (49\%) were PCR positive with a detectable viral load. Where genotyping was performed the majority were $3 \mathrm{~A}(50 \%), 1 \mathrm{~A}(22 \%)$ or $1 \mathrm{~B}(22 \%)$. The most common reasons for testing were intravenous drug use (64\%), men who have sex with men $(15 \%)$ and sexual intercourse with a known IVDU $(9 \%) .27$ patients were referred to HCV services of which only $18(67 \%)$ attended. The main reasons documented for testing within the control group were related to the patient's country of origin $(32 \%)$, country of origin of a sexual partner $(24 \%)$ or for men who have sex with men (19\%). 34 countries were given as potential sources of increased risk, however only nine patients or sexual partners of patients originated from the three countries of highest HCV prevalence.

Conclusion Sexual health services provide a unique opportunity for HCV screening in those who may otherwise not be tested. Standardisation is required to clarify who constitutes high risk particularly related to country of origin of patient or sexual partner. Initial assessment of patients diagnosed with HCV in genitourinary medicine clinics may lead to more efficient and cost effective patient care.

\section{P137 THE EFFECT OF A DEDICATED SYPHILIS CLINIC ON SYPHILIS MANAGEMENT OUTCOMES}

doi:10.1136/sextrans-2012-050601c.137

K Fernando, ${ }^{*}$ K Hall, J D C Ross. Whittall Street Clinic, Birmingham, UK

Background Patients with syphilis are frequently managed within general sexual health clinics. Although the incidence of syphilis has recently increased, it remains a relatively uncommon STI and individual clinic staff may see only a small number of cases. It is not known to what extent the use of a dedicated "syphilis clinic," where expertise is concentrated, can lead to an improvement in management. A dedicated specialist trainee/health advisor delivered syphilis clinic was introduced in April 2011 to optimise the care of patients with syphilis.

Aim To assess, using outcomes from the UK syphilis management guidelines, the effect on patient care of introducing a dedicated "syphilis clinic."

Methods A case record review of patients with syphilis before (control arm) and after (intervention arm) the introduction of a dedicated syphilis clinic between October 2010 and September 2011. Results 101 patients were analysed, 30 in the intervention, and 71 in the control arm. Repeat testing prior to treatment was undertaken in $19(63.3 \%)$ of the intervention arm and $45(63.4 \%)$ of controls $[p=0.99]$. Receipt of appropriate antibiotic treatment was observed in $28(93.3 \%)$ of the intervention arm and 65 (91.5\%) of controls $[p=0.73]$. The mean number of follow-up visits required was 1.23 (intervention arm) and 3.3 (controls) $[p=0.334]$. Of significance, however, was consultation length, being shorter in the intervention vs the control arm-mean duration of $49.7 \mathrm{~min}$ cf. $61.8 \mathrm{~min}$ $(\mathrm{p}=0.034)$.

Discussion In addition to reduced consultation duration, continuity of physician involvement and optimisation of patient care are key potential benefits of a dedicated syphilis clinic.

\section{P138 I DON'T KNOW, LET'S TRY SOME CANESTAN: AN AUDIT OF NON-SPECIFIC BALANITIS TREATMENT AND OUTCOMES}

doi:10.1136/sextrans-2012-050601c.138

E Powles, ${ }^{*}$ R Marsden, N K Gupta, T Green. Royal Hallamshire Hospital, Sheffield, UK

Background Balanoposthitis commonly presents to Genitourinary Medicine and may be caused by a wide variety of unrelated conditions. The 2008 UK National Guideline on the Management of Balanoposthitis recommends biopsy to exclude malignant disease where clinical diagnosis is uncertain and balanitis persists for more than 6 weeks despite simple treatment.

Method A retrospective audit of case notes with KC60 code C6c presenting between January and June 2011.

Results 90 patients were diagnosed with balanitis (age range 16-71 years, mean 30 , mode 22 , median 25). 68 cases were treated as presumed candidal balanitis with either Clotrimazole cream, 